\title{
1 Integrating Finite Element Modelling and 3D Printing to Engineer \\ 2 Biomimetic Polymeric Scaffolds for Tissue Engineering
}

3 Rossana Schipani ${ }^{\mathrm{a}, \mathrm{b}}$, David R. Nolan ${ }^{\mathrm{a}, \mathrm{b}}$, Caitríona Lally ${ }^{\mathrm{a}, \mathrm{b}}$ and Daniel J.

4 Kelly, a,b,d*

$5 \quad{ }^{a}$ Trinity Centre for Bioengineering, Trinity Biomedical Sciences Institute, Trinity

6 College Dublin, Dublin, Ireland;

$7 \quad{ }^{b}$ Department of Mechanical and Manufacturing Engineering, School of Engineering,

8 Trinity College Dublin, Dublin, Ireland;

$9{ }^{c}$ Department of Anatomy, Royal College of Surgeons in Ireland, Dublin, Ireland;

$10{ }^{d}$ Advanced Materials and Bioengineering Research Centre (AMBER), Royal College of

11 Surgeons in Ireland and Trinity College Dublin, Dublin, Ireland.

12 *Corresponding Author: Tel: +353-1-8963947, email: kellyd9@tcd.ie

13

14 


\section{Abstract}

16 The suitability of a scaffold for tissue engineering is determined by a number of 17 interrelated factors. The biomaterial should be biocompatible and cell instructive, with a 18 porosity and pore interconnectivity that facilitates cellular migration and the transport of 19 nutrients and waste products into and out of the scaffolds. For the engineering of load 20 bearing tissues, the scaffold may also be required to possess specific mechanical 21 properties and/or ensure the transfer of mechanical stimuli to cells to direct their 22 differentiation. Achieving these design goals is challenging, but could potentially be 23 realised by integrating computational tools such as finite element (FE) modelling with 24 three-dimensional (3D) printing techniques to assess how scaffold architecture and material properties influence the performance of the implant. In this study we first use Fused Deposition Modelling (FDM) to modulate the architecture of polycaprolactone (PCL) scaffolds, exploring the influence of varying fibre diameter, spacing and laydown 28 pattern on the structural and mechanical properties of such scaffolds. We next 29 demonstrate that a simple FE modelling strategy, which captures key aspects of the 30 printed scaffold's actual geometry and material behaviour, can be used to accurately 31 model the mechanical characteristics of such scaffolds. We then show the utility of this 32 strategy by using FE modelling to help design 3D printed scaffolds with mechanical 33 properties mimicking that of articular cartilage. In conclusion, this study demonstrates

34 that a relatively simple FE modelling approach can be used to inform the design of 3D printed scaffolds to ensure their bulk mechanical properties mimic specific target tissues.

37 Keywords: Three-dimensional Printing, Scaffold Design, Finite Element Modelling, 38 Mechanical Properties, Tissue Engineering 
40 Tissue engineering applications often require the use of porous and biocompatible three41 dimensional (3D) scaffolds that serve as temporary templates for cell attachment and 42 proliferation, ultimately promoting tissue specific extracellular-matrix secretion and 43 functional regeneration ${ }^{1,2}$. For the engineering of many tissues, the geometry and 44 mechanical properties of the scaffold are key factors that must be carefully tuned to appropriately direct regeneration. Geometrically, the scaffold needs both a suitable external architecture to properly fit the defect, and an internal architecture with sufficient porosity to facilitate cell migration and cell-cell interactions. Mechanically, it should have sufficient strength to resist physiological loading while appropriately distributing such stresses to the surrounding tissue during the regeneration process ${ }^{3}$.

3D printing technology in tissue engineering allows the fabrication of patientspecific scaffolds with high cell ingrowth capability, appropriate pore interconnectivity, highly controlled internal geometry and more recently the incorporation of bioinks containing cells ${ }^{4-6}$. Among the number of 3D printing techniques available, Fused Deposition Modelling (FDM) has shown great potential in advancing the development of functional tissue replacements, as it enables the fabrication of scaffolds with precisely defined compositions and architecture ${ }^{7,8}$. Highly interconnected pore geometries with a wide range of pores size can be obtained by varying printing parameters such as needle diameter, extrusion pressure and speed ${ }^{7-9}$. Moreover, mechanically robust scaffolds can be produced with mechanical behavior mimicking that of several biological tissues ${ }^{9-11}$. From a mechanical point of view, there is still a lack of knowledge on the behavior of 3D printed structures under compressive load and how such implants might respond to 62 physiological loading conditions. The absence of a simple and efficient framework to explain the micromechanical behavior of 3D scaffold structures can limit or slow down 
64 the development of appropriate tissue engineered scaffold designs. Previous studies attempting to develop functional scaffold designs have typically adopted a "trial-anderror" approach, where modifications to an existing design are assessed using experimental work. Computational methods that simulate the mechanical behavior of 3D constructs can also provide valuable insights into the structure-function relations of such implants ${ }^{6,12}$. A number of studies have used Finite Element Analysis (FEA) to optimize and/or evaluate scaffold designs in terms of oxygen diffusion ${ }^{13,14}$, mechanical properties ${ }^{15,16}$ and cell response to external stimuli ${ }^{13,17}$. The accuracy of such FE models strongly depends on how precisely the architecture of the printed structure is represented. Discrepancies between the originally designed structure and the actual printed geometry will always occur during the FDM process. For example, filaments from one layer of a printed scaffold fuse to differing degrees into the previous layer, altering the geometry of the scaffold. The importance of considering these geometrical differences when developing FE models of 3D printed structures has only recently been appreciated ${ }^{21,22}$. Therefore, FE analysis aided design of 3D printed scaffolds must consider the actual printed geometry of the construct, ideally without resorting to use of computationally expensive techniques that would limit the widespread use of such approaches.

The overall goal of this study was to develop a computationally efficient and accessible FE modelling strategy that could be used to design 3D printed 83 polycaprolactone (PCL) scaffolds with user-defined mechanical properties. To this end, 84 we first printed a range of PCL scaffolds with altered fibre spacing and fibre diameters, and then created Computer Aided Design (CAD)-based FE models of both the idealized scaffold designs (pre-fabrication) and the actual printed scaffolds (post-fabrication). The

87 advantage of modelling actual printed geometries and the ability of such models to predict 88 the mechanical behavior of complex $3 \mathrm{D}$ structures is demonstrated by comparing 
computational predictions to experimental measurements. The utility of this integrated approach is demonstrated by designing and 3D printing scaffolds with defined stiffness and elasticity, with a particular focus on articular cartilage tissue engineering. Computational efficiency will be ensured by using CAD-based scaffold representations, that only account for key geometrical features of the actual printed geometry parameters (e.g. fibre diameter, fibre spacing, layer fusion), to predict the mechanical behavior of 3D printed scaffolds. Using CAD-based FEA in this way is advantageous as there is no need to develop sample-specific models that require expensive and time-consuming imaging techniques (i.e. micro-computed tomography (micro-CT) to determine the geometry of the scaffolds.

\section{$100 \quad 2$ Materials and methods}

\subsection{Scaffold design and fabrication}

102 All scaffold geometries were designed to obtain cube shaped constructs with dimensions

$9 \mathrm{~mm} \times 9 \mathrm{~mm} \times 4 \mathrm{~mm}$. To investigate how geometry features can influence scaffold mechanical properties and porosity, five different architectures were obtained by varying

105 fibre spacing (s) (1 or $1.5 \mathrm{~mm}$ ), fibre diameter (d) using two different needle sizes ( 25 or

10630 Gauge) and internal fibre pattern (Aligned, Single Offset or Double Offset). Scaffold 107 geometrical features are described in Figure 1a-d. The Aligned architectures (Figure 1b) were characterized by layer X plotted orthogonally to layer X-1 (resulting in a $90^{\circ}$ angle)

109 and was plotted in the same relative position of layer X-2. The Single Offset (Figure 1c)

110 and Double Offset (Figure 1d) patterns are also orthogonal architectures characterized by

111 layer $\mathrm{X}$ being printed with an offset distance, which is half the fibre spacing, relatively to

112 the position of layer X-2. Offset layers are present only in the xz-plane for Single Offset 
113 structures (showed in red in Figure 1c), whereas they are present in both xz- and yz-

114 planes for Double Offset geometries (showed in red in Figure 1d). Fibres orientation was

115 modified after the deposition of two consecutive layers in all geometries to provide the

116 scaffolds with high side porosity. All constructs were manufactured using the 3D

117 Discovery bioplotter purchased from RegenHU (Switzerland) with spatial resolution of

$118 \pm 5 \mu \mathrm{m}$. PCL pellets with an average molecular weight (Mn) of approximately 50,000 Da

119 (CAPA 6500D, Perstorp, Sweden) were used as received. Porous PCL frames were

120 fabricated via FDM using the parameters reported in Table 1.

\subsection{Scaffold characterization}

\subsubsection{Geometry analysis}

124 The geometry of the PCL scaffolds post-printing was characterized using micro-CT.

125 Scans were performed using a Scanco Medical $40 \mu \mathrm{CT}$ system (Scanco Medical, 126 Switzerland) with a $70 \mathrm{kV}$ and $114 \mu \mathrm{A}$ x-ray source with a voxel size of $16 \mu \mathrm{m}$.

127 Simpleware ${ }^{\mathrm{TM}}$ ScanIP (Synopsys,Inc., USA) was used for processing, segmentation, 3D

128 model reconstruction and analysis of the previously obtained CT images. Scaffolds fibre 129 diameter and inter-spacing (inter-s) were measured from the top cross-sectional view of

130 the reconstructed model, while the length of two consecutive fused layers was determined

131 from the front cross-sectional view. Layer Fusion was calculated as follows:

$$
\text { Layer Fusion }(\mathrm{mm})=(2 * \text { designed } L T)-F L
$$

where LT refers to the ideally designed Layer Thickness (Figure 1) and FL indicates the

134 length of two consecutive Fused Layers in the fabricated constructs. 
137 The theoretical porosity $\left(\mathrm{P}_{\mathrm{t}}\right)$ of the designed scaffolds was estimated by volumes as 138 follows:

$$
P_{t}(\%)=1-\frac{V_{\text {scaffold }}}{V_{\text {solid }}} * 100
$$

140 where $\mathrm{V}_{\text {scaffold }}$ is the theoretical volume of the porous cubic scaffold and $\mathrm{V}_{\text {solid }}$ is the

141 volume of a non-porous cube with the same scaffold dimensions.

142 The porosity of the 3D printed structures was evaluated experimentally using the

143 gravimetric method according to the following equation:

$$
P_{e}(\%)=1-\frac{\rho_{\text {scaffold }}}{\rho_{P C L}} * 100
$$

where $\mathrm{P}_{\mathrm{e}}$ is experimental porosity, $\rho_{\text {scaffold }}$ is the apparent density of the scaffold, whereas $\rho_{\text {PCL }}$ is the PCL density which is $1.145 \mathrm{~g} / \mathrm{mL}$. $\rho_{\text {scaffold }}$ was obtained as:

$$
\rho_{\text {scaffold }}(g / m L)=\frac{m_{\text {scaffold }}}{V_{\text {scaffold }}}
$$

where $\mathrm{m}_{\text {scaffold }}$ and $\mathrm{V}_{\text {scaffold }}$ are the weight and the volume of the scaffold respectively. The weight of the 3D printed constructs was quantified using an analytical balance (Mettler Toledo Excellence XS205 DualRange with sensitivity of $0.01 \mathrm{mg}$ ).

\subsection{Mechanical characterization}

153 Mechanical tests were carried out in unconfined compression in air at room temperature

$154\left(\sim 25^{\circ} \mathrm{C}\right)$ using a twin column Zwick universal testing machine (Zwick, Roell, Germany).

155 All samples ( $\mathrm{n}=4$ per group) were subjected to a compressive-strain cycle load up to 5 156 cycles with nominal strain amplitude of $10,20,30,40$ and $50 \%$ in sequence. The

157 specimens were compressed at a cross-head speed of $1 \mathrm{~mm} / \mathrm{min}$ between two impermeable

158 metal platens after applying an initial preload of $1 \mathrm{~N}$. A 2,500 N load cell was used for 
159 testing samples produced with a 25 Gauge needle, whereas a $100 \mathrm{~N}$ load cell was used

160 for those fabricated with a 30 Gauge needle. The load versus displacement data were

161 recorded throughout. The engineering stress and strain were calculated by dividing the

162 load value with the initial apparent cross-sectional area of each sample and the

163 displacement value with the initial sample height, respectively. The elastic modulus was

164 taken as the slope of the initial linear region of the plotted stress-strain curve obtained

165 from the first compressive cycle.

166 The scaffold permanent deformation (PD), defined as apparent uniaxial plastic strain in

167 the material, was calculated at the end of the tests as follows:

168

$$
P D(\%)=\frac{\text { Test Speed } * \Delta t_{5}}{h_{0}} * 100
$$

where $\Delta \mathrm{t}_{5}(\mathrm{~s})$ is the interval of time at the start of the $5^{\text {th }}$ cycle in which no force is applied, assuming the sample underwent permanent deformation, while $\mathrm{h}_{0}(\mathrm{~mm})$ is the height of the sample prior to test.

\subsection{Finite element analysis}

174 To predict the compressive properties of the 3D printed scaffolds, CAD-based FE models

175 were developed using ABAQUS v6.14 (DS Simulia, USA). For Aligned $1(d=0.3 \mathrm{~mm}$;

$176 s=1.0 \mathrm{~mm})$ and Aligned $2(d=0.3 \mathrm{~mm} ; s=1.5 \mathrm{~mm})$ structures, ramp compression tests until

$17710 \%$ strain were simulated for both an idealized and an actual printed scaffold

178 representation. In the idealized models, pre-fabrication scaffold geometry features were

179 reproduced. On the other hand, actual printed scaffold models were characterized by

180 geometry parameters measured post-fabrication including layer fusion. For Aligned 3

$181 \quad(d=0.12 \mathrm{~mm} ; s=1.5 \mathrm{~mm})$, Single Offset $(d=0.12 \mathrm{~mm} ; s=1.5 \mathrm{~mm})$ and Double Offset

$182(d=0.12 \mathrm{~mm} ; s=1.5 \mathrm{~mm})$ structures, only actual printed models were developed simulating 
183 the same mechanical loading conditions as for Aligned 1 and Aligned 2. Both idealized

184 and actual printed models consisted of a symmetric structure of approximately cubic

185 scaffolds $(4.5 \mathrm{~mm} \times 4.5 \mathrm{~mm} \times 4 \mathrm{~mm})$. For all groups, the nodes at the top face of the

186 scaffolds were given a displacement of approximately $0.4 \mathrm{~mm}$ corresponding to $10 \%$

187 compressive strain. The nodes at the bottom ends of the constructs were constrained only

188 in the direction of loading, allowing for scaffold expansion in the remaining two

189 directions due to the Poisson's effect. Symmetry boundary conditions were also applied

190 as the model was reduced to a quarter section cut along the $\mathrm{xz}$ and yz planes of symmetry.

191 Therefore, $\mathrm{x}$ and $\mathrm{y}$ DOFs perpendicular to the symmetry planes were constrained.

192 The effective compressive modulus of the constructs was determined from the stress and

193 strain values of the linear region of the curve calculated from the displacement and

194 resultant reaction force data computed from the simulations. To compare the predictions

195 to the experiments, the resultant reaction force was multiplied by four to evaluate the

196 models outputs for the entire constructs. Isotropic elastic behaviour was initially assumed

197 for Aligned 1 and Aligned 2 models. Quadratic ten-node tetrahedral elements (C3D10)

198 were used. Table 3 summarizes the material properties of PCL which were obtained from

199 literature ${ }^{19,23,24}$.

\subsubsection{Elastoplastic material model}

201 As PCL will deform plastically once the stress in the material exceeds its yield stress, an

202 elastoplastic material model is preferable to an elastic material model when the stress in

203 the material is expected to exceed the yield stress during loading ${ }^{25,26}$. To predict more

204 accurately the PCL scaffolds' stress-strain behaviour under compression, uniaxial

205 elastoplastic models were implemented (only for the actual printed geometries for all

206 scaffold groups). The same model configuration and boundary conditions as in the purely 
207 elastic material models were applied. The plasticity model used was the von Mises yield 208 criterion with isotropic hardening. To define the stress-strain curve, the yield and failure

209 points of the material were considered as found in literature ${ }^{23}$. The implemented material

210 parameters are summarized in Table 3. In Abaqus the plastic input parameters required

211 were true stress and true plastic strain. Assuming no volume change in the specimen, the

212 true stress $\left(\sigma_{\text {true }}\right)$ was calculated as follows:

$$
\sigma_{\text {true }}=\sigma_{\text {eng }} *\left(1+\varepsilon_{\text {eng }}\right)
$$

214 where $\sigma_{\text {eng }}$ and $\varepsilon_{\text {eng }}$ are engineering stress and strain.

215 The true total strain $\left(\varepsilon_{\text {true }}\right)$ was calculated as:

216

$$
\varepsilon_{\text {true }}=\ln \left(1+\varepsilon_{\text {eng }}\right)
$$

217 from which the true plastic strain $\left(\varepsilon_{\mathrm{pl}}\right)$ was obtained as:

$$
\varepsilon_{p l}=\varepsilon_{\text {true }}-\varepsilon_{e l}=\varepsilon_{\text {true }}-\left(\frac{\sigma_{\text {true }}^{y}}{E}\right)
$$

219 where $\varepsilon_{\mathrm{el}}$ is the elastic strain, $\sigma_{\text {true }}^{y}$ is the true yield stress and E is the Young's modulus.

\subsubsection{Determination of permanently deformed element volume fraction}

221 To determine theoretically which scaffold architecture was more likely to undergo higher permanent deformation, the element volume fraction experiencing stress greater than 17

$223 \mathrm{MPa}$, which is approximately the yield stress of PCL ${ }^{23,24}$, was quantified as follows:

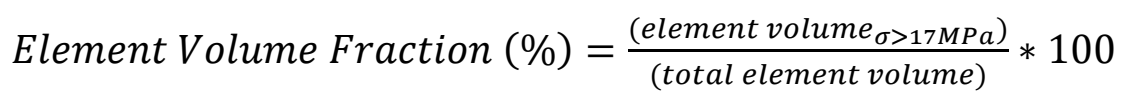


where element volume ${ }_{\sigma}>17 \mathrm{MPa}$ represents the volume of the elements in the FE model

226 showing stress greater than $17 \mathrm{MPa}$, whereas total element volume represents the volume

227 of all the elements composing the scaffold model.

\subsection{Statistical Analysis}

229 Statistical analysis was performed using GraphPad (GraphPad Software, La Jolla

230 California USA). Compressive modulus, porosity and permanent deformation analysis

231 for varying filament spacing (Aligned 1 vs. Aligned 2) and filament diameter (Aligned 2

232 vs. Aligned 3) were examined using a student's t-test where means were compared.

233 One-way analysis of variance (ANOVA) with the addition of Tukey's correction was

234 used for multiple comparisons testing (Aligned 3 vs. Single Offset vs. Double Offset).

235 Results are expressed as mean \pm standard deviation. For all comparisons, the level of 236 significance was $\mathrm{p} \leq 0.05$.

\section{Results}

\subsection{The effect of filament diameter and spacing on the porosity and mechanical} properties of $3 D$ printed PCL scaffolds

241 To evaluate the effect of filament diameter and spacing on both the porosity and

242 mechanical properties of 3D printed PCL scaffolds, three different idealized architectures

243 were designed as shown in Figure 1b. The designed constructs were characterized by a

244 fibrous network comprising of aligned filaments stacked in horizontal layers that

245 followed a $0^{\circ} 0^{\circ}-90^{\circ} 90^{\circ}$ pattern. Aligned 1 and Aligned 2 have a filament diameter of

$2460.26 \mathrm{~mm}$ (25 gauge needle) and two different spacings, 1 and $1.5 \mathrm{~mm}$ respectively. This

247 resulted in filament inter-spacings of $0.74 \mathrm{~mm}$ for Aligned 1 and $1.24 \mathrm{~mm}$ for Aligned 2. 
248 To study the effect of filament diameter, Aligned 3 architecture had a fibre diameter of $2490.16 \mathrm{~mm}$ (30 gauge needle), while the fibre spacing was the same as the Aligned 2 design $250(1.5 \mathrm{~mm})$, resulting in a fibre inter-spacing of $1.34 \mathrm{~mm}$.

The actual printed structures of the different scaffold designs are shown in Figure 2. 3D printing allowed for accurate and controlled deposition of PCL filaments, although micro-CT reconstructions demonstrate that some fibre diameter inhomogeneities exist in all three structures (Figure 2a-c). From the CT scans, the average fibre diameter was found to be approximately $0.3 \mathrm{~mm}$ in both the Aligned 1 and Aligned 2 architectures, whereas the filament diameter was about $0.12 \mathrm{~mm}$ for Aligned 3. Therefore, the interspacing between consecutive struts was smaller in Aligned $1(0.660 \pm 0.017 \mathrm{~mm})$ and Aligned $2(1.168 \pm 0.089 \mathrm{~mm})$ compared to the ideal designs, while it was bigger in Aligned $3(1.373 \pm 0.025 \mathrm{~mm})$. This had an effect on the resultant porosity of the actual printed scaffolds (Table 2). Compared to the idealized structures, Aligned 1 and Aligned 2 structures were less porous, whereas Aligned 3 scaffolds had greater porosity. From

262 the cross-sections of the CT scan images (Figure 2), it was observed that the printed 263 filaments in all architectures did not have a regular rounded shape as ideally designed.

264 This is because some degree of fusion between consecutively deposited layers occurred. 265 Layer Fusion, which is considered 0 in the ideal designs, was quantified according to 266 equation (1). It was found to be approximately $0.08 \mathrm{~mm}$ in Aligned 1 and Aligned 2, 267 whereas it was approximately $0.02 \mathrm{~mm}$ in Aligned 3 (Table 2).

269 The compressive modulus and the extent of permanent deformation following the 270 application of cyclic strain was calculated for each scaffold design (Figure 3a). 271 Representative stress-strain plots of the first loading cycle for the three architectures are 
272 shown in Figure 3b. As expected, increasing the filament fibre spacing from $1 \mathrm{~mm}$

273 (Aligned 1) to $1.5 \mathrm{~mm}$ (Aligned 2) increased the porosity and reduced the compressive

274 modulus of the resulting scaffold (Table 2; Figure 3c). Both Aligned 1 and Aligned 2

275 geometries experienced permanent deformation after the application of the first

276 compressive cycle (10 \% applied strain) as it is shown in Supplementary Figure 1a,b for

277 Aligned 1 and Aligned 2 scaffolds, respectively. Overall, the higher porosity scaffolds

278 (Aligned 2) underwent higher permanent deformation $(\sim 25 \%)$ compared to the less

279 porous constructs (Aligned 1; 22\%) (Figure e). Reducing the filament diameter (Aligned

280 3) also increased the porosity and reduced the compressive modulus of the scaffold (Table

281 2; Figure 3d). Moreover, lower permanent deformation was observed (Figure 3f).

\subsection{FE models incorporating actual printed geometries can accurately predict} the mechanical behaviour of $3 D$ printed scaffolds

FE simulations of unconfined ramp compression tests were first performed using both idealized and actual printed geometries for the Aligned 1 (Figure 4a,c) and Aligned 2

287 (Figure 4b,d) structures using an elastic material model. In idealized in silico models, the ideally designed geometry parameters generated by CAD models were used to represent the constructs. In actual printed models, scaffolds were reproduced using the structural features measured post-fabrication where the actual fibre diameter and the amount of fusion between layers was included as model parameters. The von Mises stress was

292 predicted to be higher at the crossover areas between consecutive printed layers for both idealized (Figure 4a,b) and actual printed (Figure 4c,d) model for the Aligned 1 (Figure 4a,c) and Aligned 2 (Figure 4b,d) scaffolds. Comparing the predicted stress-strain behaviour with the experimental results (Figure 5a,c), it can be observed that using the idealized representation of both Aligned 1 (Figure 5a) and Aligned 2 (Figure 5c) scaffolds 
297 resulted into a significant underestimation of the bulk compressive modulus (Figure

$2985 \mathrm{~b}, \mathrm{~d})$. On the other hand, the actual printed models, which reproduced key scaffold

299 geometrical features (including layer fusion) as they were measured after fabrication,

300 showed good agreement with the experimental measurement of compressive modulus for

301 the Aligned 1 (Figure 5b) and Aligned 2 (Figure 5d) scaffolds. Nevertheless, actual printed

302 models that considered only elastic material properties failed to accurately predict the

303 stress-strain response, specifically the apparent transition from the linear elastic to the

304 plastic region under compression (Figure 5a,c).

305 Due to the architecture of these scaffolds it is expected that some local permanent

306 deformation will occur within the body of the scaffold once the localized stress exceeded

307 the material yield stress (the yield stress of PCL is estimated to be $\sim 17 \mathrm{MPa}{ }^{23,24}$ ).

308 Therefore, an elastoplastic material model for PCL was introduced and simulations of

309 ramp compression tests were performed only for the actual printed configurations of both

310 Aligned 1 (Figure 4e) and Aligned 2 (Figure 4f). The predicted peak values of von Mises

311 stress were lower using the elastoplastic material model (Figure 5a,c). Furthermore, the

312 predicted stress-strain behaviour was more representative of experimental observations.

313 To evaluate the effect of varying filament diameter on scaffold mechanical

314 properties using FEA, in silico models of the Aligned 3 constructs were also developed

315 using an elastoplastic material model and the actual printed geometry (Figure 6d-f). When

316 comparing Aligned 2 and Aligned 3 models, it can be observed once again that the

317 compressive forces are mainly supported at the filament junctions of adjacent layers,

318 although the stresses generated within the Aligned 3 structure were lower compared to

319 Aligned 2 (Figure 6a,d). The actual printed elastoplastic models were again capable of

320 accurately predicting the stress-strain behaviour (Figure 6e) and compressive modulus

321 (Figure 6f) of the scaffolds. 


\subsection{FE modelling to inform the design of 3D printed scaffolds with user defined} mechanical properties

325 Having developed a computational framework that was able to accurately predict the uniaxial compression behaviour of 3D printed scaffolds, we next sought to leverage this approach to design scaffolds with biomimetic mechanical properties. Articular cartilage has a region specific-compressive modulus that varies from approximately $0.25 \mathrm{MPa}$ to 1.8 $\mathrm{MPa}^{27,28}$. Ideally scaffolds designed to regenerate this tissue should have mechanical

330 properties falling in the aforementioned range to provide a physiological-like mechanical environment.

The effect of varying filament pattern of fibrous constructs was evaluated. Figure $1 \mathrm{c}, \mathrm{d}$ shows the strategies adopted to modify the scaffold fibre arrangement starting from the Aligned 3 structure. The new designed architectures had the same filament diameter and spacing as Aligned 3, but different filament pattern. The Single Offset architecture (Figure 1c) was characterized by offset layers present only in the xz-plane, whereas it showed a regular orthogonal arrangement in the yz-plane. The Double Offset scaffold (Figure 1d) differed from the previous one because it had offset layers in both xz- and yzplanes. The offset was set to $0.75 \mathrm{~mm}$ (half the fibre spacing) in both cases. Single Offset and Double Offset mechanical properties were predicted simulating compression tests as

341 done previously. Von Mises stress plots for the Single Offset architecture (Figure 7a) were

342 similar to the previously analysed structures if looking at the yz-plane where filaments 343 are arranged orthogonally with no offset. Here higher levels of stress were experienced 344 at the points where filaments crossed over. On the xz-plane, the stress was not particularly 345 concentrated in certain areas, but it was more homogeneously distributed through the 
fibres.

In the Double Offset architecture model (Figure 7b), the same homogeneous stress contour, which was present only in the xz-plane for the Single Offset model, was observed throughout. This resulted in overall lower levels of stress experienced by the Double Offset architecture. Predicted compressive stress-strain curves (Figure 7c) showed that varying the filament pattern of the porous scaffolds from Aligned 3 to Single Offset and Double Offset decreased the stiffness of the constructs. This was confirmed when calculating the compressive modulus (Figure 7d) which was 1.88, 0.56 and $0.22 \mathrm{MPa}$ for Aligned 3, Single Offset and Double Offset respectively.

To predict which architecture is more likely to undergo higher permanent deformation, the element volume fraction of each model which experienced stress greater than $17 \mathrm{MPa}$ (PCL yield stress) was calculated according to equation (9). Figure 7e shows the quantified element volume fraction for the three analysed models. It was predicted that the Aligned 3 configuration had the highest volume fraction $(9.56 \%)$ indicating this was the structure that would experience more permanent deformation when subjected to $10 \%$ compression. The Single Offset model had a volume fraction of $4.44 \%$ and the Double Offset model had $0.2 \%$, thereby the latter having the lowest plastic deformation.

\subsubsection{Models validation}

Single Offset and Double Offset PCL constructs were 3D printed according to the fabrication parameters used for the Aligned 3 architecture as reported in Table 1. Figure

366 8a shows microscope images of the obtained scaffolds. Constructs were mechanically tested following the same cyclic compression test protocol applied for the previous experiments. Representative stress-strain curves of the first loading cycle are shown in

369 Figure 8b, in which Aligned 3, Single Offset and Double Offset mechanical properties are 
compared. As was predicted, Double Offset constructs are the softest whereas Aligned 3

371 structures are the stiffest among the three groups. This was evidenced by the differences

372 in slope of the stress-strain curves. Moreover, the trend of the experimental curves

373 matched the predicted ones. The fabricated scaffolds where characterized by high

374 porosity which was about $90 \%$ regardless of the filament pattern chosen (Figure 8c,d).

375 In good agreement with the computational results, the Single Offset and Double Offset

376 constructs had a compressive modulus of $0.817 \pm 0.02 \mathrm{MPa}$ and $0.320 \pm 0.03 \mathrm{MPa}$

377 respectively (Figure 8c). Furthermore, varying the arrangement of the scaffold filaments

378 reduced the permanent deformation the constructs underwent after being subjected to

379 cyclic compressive loadings. All scaffold geometries underwent plastic deformation after

380 being subjected to $10 \%$ compressive strain (Supplementary Figure 1c-e). Permanent

381 deformation at the end of the test was measured to decrease from about $18 \%$ in Aligned

3823 structures to approximately 16 and $14 \%$ in Single Offset and Double Offset constructs

383 respectively (Figure 8d). Once again, CAD-FE models based on actual printed scaffold

384 geometry proved to be an efficient approach to design constructs with desired structural

385 and mechanical properties.

\section{Discussion}

388 The fabrication of scaffolds with a controlled shape and interconnected pore network, as

389 well as appropriate mechanical properties, is fundamental when developing tissue

390 engineered constructs $1,2,10,29,30$. 3D printing allows such control and permits the creation

391 of constructs that serve as temporary templates while the extracellular matrix is produced,

392 and can provide a mechanical environment conductive to tissue formation, especially

393 when combined with soft hydrogel materials ${ }^{31-35}$. Computational modelling has been 
394 increasingly applied to tissue engineering in order to aid in the design of such 3D

395 scaffolds ${ }^{36-39}$. However, it can be challenging to develop FE models capable of 396 accurately predicting scaffolds mechanical properties, at least in part due to unintended 397 geometrical differences between the idealized and actual fabricated scaffolds ${ }^{3,40,41}$. 398 Herein we described a strategy for designing 3D printed scaffolds with different structural 399 and mechanical properties that is informed by a FE model that accounts for differences 400 between the idealized scaffold geometry and what is eventually printed. Models of 401 different scaffold architectures provided an insight into the structure-function relation of 402 such scaffolds, and how modifying specific structural features can tailor the mechanical 403 properties to those of a wide range of native tissues.

404 Using FDM, we produced a number of scaffolds made of PCL, which is a synthetic 405 polymer widely used in 3D printing due to its biocompatibility, low melting temperature 406 and mechanical stability $9,31,42,43$. The optimal sets of fabrication parameters for two 407 different needle sizes (25 and 30 Gauge) were chosen to obtain defined porous structures 408 with a good resolution and to avoid delamination between consecutively printed layers.

409 Varying PCL scaffold geometrical features such as filament spacing and diameter had an 410 effect on scaffold porosity, mechanical properties and plastic deformation. Increasing the 411 fibre spacing from $1 \mathrm{~mm}$ (Aligned 1) to $1.5 \mathrm{~mm}$ (Aligned 2), but maintaining the same 412 fibre diameter, resulted in structures with a higher porosity and therefore a lower 413 compressive modulus. The more porous scaffolds also experienced higher permanent 414 deformation. This may be due to sagging of the filaments when spanning from one fibre 415 to the next, resulting in densification (impacting of the fibres against one another) of the 416 scaffold occurring earlier when compression forces are applied. Scaffold stiffness further 417 decreased whereas porosity increased when reducing fibre diameter (Aligned 3), although 418 lower permeant deformation was observed. This is likely due to the lower stresses (and 
419 hence material yielding) that are predicted to be generated within the scaffolds with lower

420 fibre diameters as they are compressed.

421 Using micro-CT, we revealed geometric discrepancies between idealized and actual

422 printed structures which are dependent on the fabrication process. Depending on the set

423 of fabrication parameters used, the PCL filament diameter was either larger (for the 25

424 Gauge needle) or smaller (for the 30 Gauge needle) than originally designed. Moreover,

425 the shape of the individual fibres was hard to distinguish as consecutively printed layers

426 fused together post-extrusion. Such discrepancies impact both scaffold geometry and

427 mechanical properties ${ }^{8,44,45}$, but to date there are only few modelling techniques that have

428 simulated these geometrical variations which have mainly focused on scaffolds for the 429 regeneration of hard tissues ${ }^{21,22,46-49}$. For example, Campoli et al. ${ }^{47}$ utilized FE models

430 that implemented variations in the cross-section area of the struts in porous metallic

431 biomaterials, showing good predictions when comparing computational and experimental

432 results. Melancon et al. ${ }^{48}$ developed a morphological map that would capture structural

433 differences post-fabrication of porous biomaterials, which was then used to create

434 statistical based numerical models that incorporated such geometrical irregularities.

435 These models produced more reliable predictions of experimentally measured mechanical

436 properties. Ravari et al. ${ }^{49}$ developed a strategy to take account of variations in filament

437 diameter into their $\mathrm{FE}$ models of 3D printed structures, which also improved the

438 predictive capacity of the computational models. Naghieh et al. ${ }^{22,46}$ investigated the effect

439 of fusion between the different layers in 3D printed scaffolds, and again demonstrated the

440 importance of considering this when developing accurate FE models. In the current study,

441 a FE modelling framework was used to design scaffolds with mechanical properties

442 suitable for soft tissue applications. CAD-based FE models of the idealized and actual

443 printed scaffold architectures were developed to study the impact of such geometrical 
444 differences when predicting the scaffold mechanical properties. Our models

445 demonstrated that including layer fusion is essential to accurately modelling 3D printed

446 scaffolds. Indeed, when comparing idealized to actual printed models (Figure 4 and 5),

447 we have shown that the idealized model, in which layer fusion was not accounted for,

448 was not able to provide accurate predictions because the geometry of the scaffold itself

449 (e.g. scaffold height) was inaccurate. The actual printed models described filament

450 diameter and amount of layer fusion as measured post-fabrication. For both Aligned 1 and

451 Aligned 2 designs, modelling the idealized structures lead to a significant underestimation

452 of the mechanical properties compared to the experimental results. On the other hand, the

453 predicted compressive stiffness of the actual printed designs showed good agreement with

454 the experiments, especially when the plasticity of PCL was also considered.

455 Implementing an elastoplastic material model not only accurately predicted the

456 compressive elastic modulus of the 3D printed constructs but also captured the

457 mechanical behaviour past the yield point. This was observed for Aligned 1, Aligned 2

458 and Aligned 3 scaffold models.

459 To demonstrate how the proposed computational approach could be used to help inform

460 the design of a scaffold prior to printing, the laydown filament pattern of the actual printed

461 Aligned 3 structure was theoretically modified to obtain Single Offset and Double Offset

462 architectures. Introducing offset layers in one plane only (Single Offset) or in two planes

463 (Double Offset) reduced the compressive stiffness by almost one order of magnitude

464 (compressive modulus was decreased from 1.88 to 0.56 and ultimately to $0.22 \mathrm{MPa}$ for

465 Aligned 3, Single Offset and Double Offset designs, respectively), despite the scaffold 466 porosity being maintained constant. Varying the filament pattern also reduced the

467 permanent deformation within the scaffolds following the application of a defined level 468 of compressive strain. In the stiffer Aligned scaffolds, deformation of the entire scaffold 
primary occurs due to the filaments undergoing compressive strain. The scaffold is better designed to resist compressive deformation as columns of material are generated where

471 filament layers overlap, and large strains and stresses are generated locally in the scaffold

472 material at these points of overlap (Figure 4 and 6a, d). These large local stresses cause

473 the material to locally undergo plastic deformation. In the softer Offset scaffolds,

474 deformation of the scaffold occurs due to bending of the filaments. As the scaffold 475 deforms in this way, it offers less resistance to compressive loading and smaller stresses and strains are generated locally within the scaffold material (Figure 7a, b); such smaller local stresses result in lower levels of permanent deformation. Experimental compression tests confirmed the ability of the FE modelling framework to produce scaffolds with specific mechanical attributes prior to their fabrication. Experimentally, the porosity of the analysed structures was the same and the compressive moduli matched the predicted values. In summary, we have developed a computationally efficient modelling approach using CAD-based scaffold representations that account for key geometrical features of the actual printed construct to predict the mechanical behaviour of 3D printed scaffolds.

484 Employing such CAD-based FE models by using the average values of the scaffold geometrical parameters measured experimentally is advantageous as there is no need to develop computationally expensive sample-specific models that require complex and time-consuming imaging techniques (i.e. micro-CT) to accurately determine the geometry of the scaffolds. This approach is particularly beneficial in the initial scaffold 489 design phase, although considering sample-specific geometries (which we have not 490 undertaken in this study) will be important if trying to understand the variability in scaffold mechanical properties from print to print. 


\section{Conclusion}

494 This study demonstrates the benefits of combining computational and experimental 495 strategies for engineering spatially complex scaffolds. Specifically, a simple and 496 relatively accessible FE strategy was developed, which was shown capable of 497 successfully predicting the mechanical properties of 3D printed scaffolds prior to their 498 fabrication. The geometric discrepancies between scaffold designs pre- and post499 fabrication was found to be critical in developing FE models capable of accurately 500 predicting the mechanical behaviour of 3D printed scaffolds. A number of strategies to 501 modulate the structural and mechanical properties of 3D printed PCL scaffolds was 502 explored, allowing constructs to be obtained with compressive properties spanning from 503 the $\mathrm{kPa}$ to the MPa range. Thus, the proposed FEA method combined with 3D printing 504 represents a powerful approach to producing biomaterial scaffolds mimicking the 505 mechanical properties of a broad range of biological tissues.

\section{$507 \quad$ Funding}

508 This work was supported by the European Research Council (JointPrint; ERC-CoG509 2014-647004).

\section{Disclosure statement}

512 No potential conflict of interest was reported by the authors

\section{References}

515 1. Brien, F. J. O. Biomaterials and scaffolds for tissue engineering. Mater. Today

$516 \quad \mathbf{1 4},(2011)$.

517 2. Hollister, S. J. Porous scaffold design for tissue engineering. Nat. Mater. 4, 518- 
519 3. Lohfeld, S., Cahill, S., Doyle, H. \& McHugh, P. E. Improving the finite element model accuracy of tissue engineering scaffolds produced by selective laser sintering. J. Mater. Sci. Mater. Med. 26, 1-12 (2015).

4. Park, J. S. et al. The effect of matrix stiffness on the differentiation of mesenchymal stem cells in response to TGF- $\beta$. Biomaterials 32, 3921-30 (2011).

5. Murphy, S. V. \& Atala, A. 3D bioprinting of tissues and organs. Nat. Biotechnol. 32, 773-785 (2014).

6. Giannitelli, S. M., Accoto, D., Trombetta, M. \& Rainer, A. Current trends in the design of scaffolds for computer-aided tissue engineering. Acta Biomater. 10, 580-594 (2014).

529 7. Sachlos, E., Czernuszka, J. T., Gogolewski, S. \& Dalby, M. Making tissue engineering scaffolds work. Review on the application ofsolid freeform fabrication technology to the production of tissue engineeringscaffolds. Eur.

8. Zein, I., Hutmacher, D. W., Tan, K. C. \& Teoh, S. H. Fused deposition modeling of novel scaffold architectures for tissue engineering applications. Biomaterials 23, 1169-1185 (2002).

9. Olubamiji, A. D. et al. Modulating mechanical behaviour of 3D-printed cartilagemimetic PCL scaffolds: influence of molecular weight and pore geometry. Biofabrication 8, 025020 (2016).

10. Woodfield, T. B. F. et al. Design of porous scaffolds for cartilage tissue engineering using a three-dimensional fiber-deposition technique. Biomaterials 25, 4149-4161 (2004).

542 11. Roohani-Esfahani, S.-I., Newman, P. \& Zreiqat, H. Design and Fabrication of 3D 
printed Scaffolds with a Mechanical Strength Comparable to Cortical Bone to Repair Large Bone Defects. Sci. Rep. 6, 19468 (2016).

12. Lacroix, D., Planell, J. A. \& Prendergast, P. J. Computer-aided design and finiteelement modelling of biomaterial scaffolds for bone tissue engineering. Philos.

13. O'Reilly, A. \& Kelly, D. J. Unravelling the Role of Mechanical Stimuli in Regulating Cell Fate During Osteochondral Defect Repair. Ann. Biomed. Eng. 44, 3446-3459 (2016).

14. Woo Jung, J. et al. Evaluation of the effective diffusivity of a freeform fabricated

15. Eshraghi, S. \& Das, S. Micromechanical finite-element modeling and experimental characterization of the compressive mechanical properties of polycaprolactone-hydroxyapatite composite scaffolds prepared by selective laser

16. Almeida, H. A. \& Bártolo, P. J. Numerical simulations of bioextruded polymer sintering for bone tissue engineering. Acta Biomater. 8, 3138-3143 (2012). scaffolds for tissue engineering applications. Polym. Int. 62, 1544-1552 (2013).

17. Yan, K. C., Nair, K. \& Sun, W. Three dimensional multi-scale modelling and analysis of cell damage in cell-encapsulated alginate constructs. J. Biomech. 43,

19. Lohfeld, S., Cahill, S., Doyle, H. \& McHugh, P. E. Improving the finite element model accuracy of tissue engineering scaffolds produced by selective laser 
20. Campos Marin, A. \& Lacroix, D. The inter-sample structural variability of regular tissue-engineered scaffolds significantly affects the micromechanical local cell environment. Interface Focus 5, 20140097-20140097 (2015).

21. Gleadall, A., Ashcroft, I. \& Segal, J. VOLCO: A predictive model for 3D printed microarchitecture. Addit. Manuf. 21, 605-618 (2018).

22. Naghieh, S. et al. Modeling of the Mechanical Behavior of 3D Bioplotted Scaffolds Considering the Penetration in Interlocked Strands. Appl. Sci. 8, 1422 (2018).

23. Rosa, D. S. et al. Evaluation of the Thermal and Mechanical Properties of and Their Blends. J. Appl. Polym. Sci. 91, 3909-3914 (2004).

24. Ragaert, K. \& Cardon, L. Bulk mechanical properties of thermoplastic poly- $\varepsilon-$ caprolactone. J. Mater. Sci. Mater. Med. 20, 1255-62 (2009).

25. Ribeiro, J. F. M. et al. Structural monitoring and modeling of the mechanical deformation of three-dimensional printed poly( $\varepsilon$-caprolactone) scaffolds.

26. Entezari, A. et al. Yielding behaviors of polymeric scaffolds with implications to tissue engineering. Mater. Lett. 184, 108-111 (2016).

27. Boschetti, F., Pennati, G., Gervaso, F., Peretti, G. M. \& Dubini, G. Orthop. Res. 12, 340-349 (1994).

29. Salerno, A., Oliviero, M., Di Maio, E., Iannace, S. \& Netti, P. A. Design of 
Sci. Mater. Med. 20, 2043-2051 (2009).

594 30. Bracaglia, L. G. et al. 3D printing for the design and fabrication of polymerbased gradient scaffolds. Acta Biomater. 56, 3-13 (2017).

596

31. Critchley, S. E. \& Kelly, D. J. Bioinks for bioprinting functional meniscus and articular cartilage. J. 3D Print. Med. 1, 269-290 (2017).

32. Daly, A. C. et al. 3D Bioprinting of Developmentally Inspired Templates for Whole Bone Organ Engineering. Adv. Healthc. Mater. 5, 2353-62 (2016).

33. Liao, I.-C., Moutos, F. T., Estes, B. T., Zhao, X. \& Guilak, F. Composite ThreeCreate Functional Synthetic Articular Cartilage. Adv. Funct. Mater. 23, 58335839 (2013).

34. Castilho, M. et al. Mechanical behavior of a soft hydrogel reinforced with threedimensional printed microfibre scaffolds. Sci. Rep. 8, 1245 (2018).

35. Bas, O. et al. Biofabricated soft network composites for cartilage tissue engineering. Biofabrication 9, (2017).

36. Milan, J. L., Planell, J. A. \& Lacroix, D. Computational modelling of the mechanical environment of osteogenesis within a polylactic acid-calcium phosphate glass scaffold. Biomaterials 30, 4219-4226 (2009).

611 37. Hendrikson, W. J., van Blitterswijk, C. A., Rouwkema, J. \& Moroni, L. The Use of Finite Element Analyses to Design and Fabricate Three-Dimensional Scaffolds for Skeletal Tissue Engineering. Front. Bioeng. Biotechnol. 5, 1-13 (2017).

614 38. Moroni, L. et al. Finite Element Analysis of Meniscal Anatomical 3D Scaffolds: Implications for Tissue Engineering. Open Biomed. Eng. J. 1, 23-34 (2007). 
Appl. Mater. Interfaces 9, 29430-29437 (2017).

619

620

621

622

623

624

625

626

627

628

629

630

631

632

633

634

635

636

637

638

639

640

641

642

40. Cahill, S., Lohfeld, S. \& McHugh, P. E. Finite element predictions compared to experimental results for the effective modulus of bone tissue engineering scaffolds fabricated by selective laser sintering. J. Mater. Sci. Mater. Med. 20, 1255-1262 (2009).

41. Doyle, H., Lohfeld, S., McDonnell, P. \& McHugh, P. Evaluation of a Multiscale Modelling Methodology to Predict the Mechanical Properties of PCL/ $\beta$-TCP Sintered Scaffold Materials. Ann. Biomed. Eng. 43, 1989-1998 (2014).

42. Daly, A. C. et al. 3D Bioprinting for Cartilage and Osteochondral Tissue Engineering. Adv. Healthc. Mater. 1700298, 1700298 (2017).

43. Woodruff, M. A. \& Hutmacher, D. W. The return of a forgotten polymer Polycaprolactone in the 21st century. Prog. Polym. Sci. 35, 1217-1256 (2010).

44. Tellis, B. C. et al. Trabecular scaffolds created using micro CT guided fused deposition modeling. Mater. Sci. Eng. C 28, 171-178 (2008).

45. Domingos, M. et al. Effect of process parameters on the morphological and mechanical properties of 3D Bioextruded poly(1-caprolactone) scaffolds. Rapid Prototyp. J. 18, 56-67 (2012).

46. Naghieh, S., Karamooz Ravari, M. R., Badrossamay, M., Foroozmehr, E. \& Kadkhodaei, M. Numerical investigation of the mechanical properties of the additive manufactured bone scaffolds fabricated by FDM: The effect of layer penetration and post-heating. J. Mech. Behav. Biomed. Mater. 59, 241-250 (2016).

47. Campoli, G. et al. Mechanical properties of open-cell metallic biomaterials manufactured using additive manufacturing. Mater. Des. 49, 957-965 (2013).

48. Melancon, D. et al. Mechanical characterization of structurally porous 
649

650

651

652

653

654

655

656

657

658

659

660

661

662

663

664

665

666

667

biomaterials built via additive manufacturing: experiments, predictive models, and design maps for load-bearing bone replacement implants. Acta Biomater. 63, 350-368 (2017).

49. Karamooz Ravari, M. R., Kadkhodaei, M., Badrossamay, M. \& Rezaei, R. Numerical investigation on mechanical properties of cellular lattice structures fabricated by fused deposition modeling. Int. J. Mech. Sci. 88, 154-161 (2014).

(1)


a
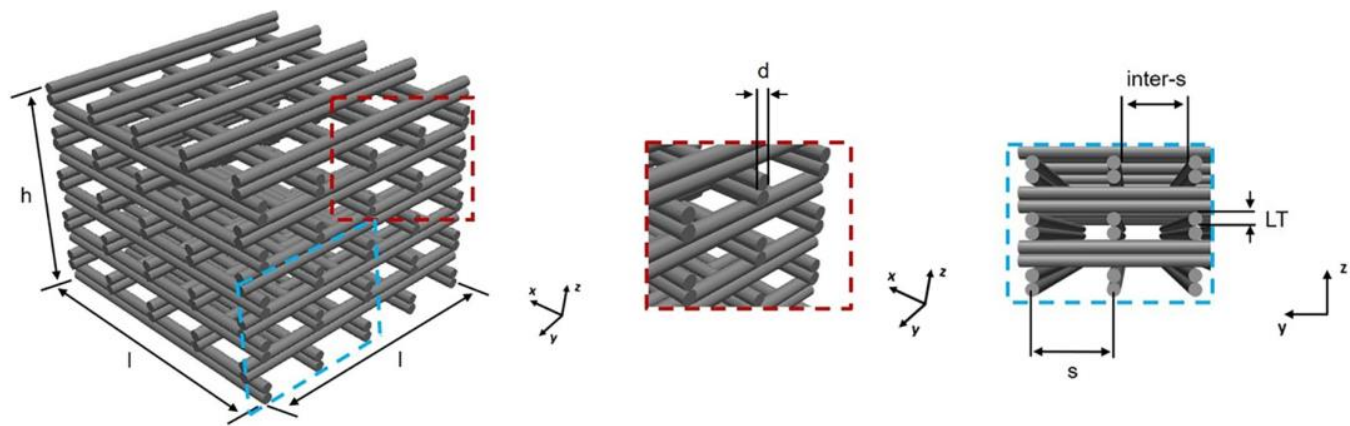

b

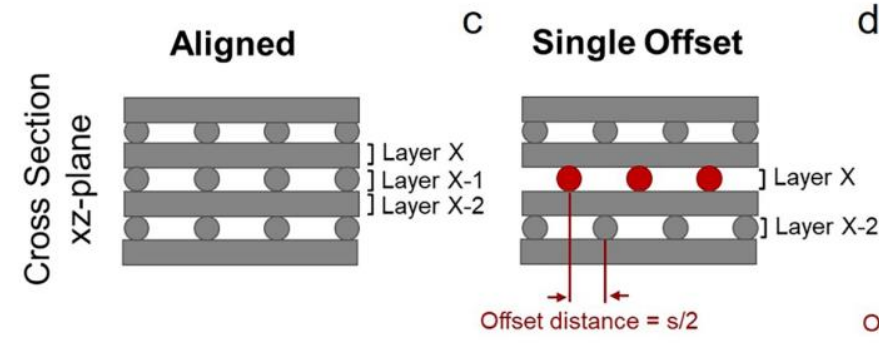

d Double Offset

668
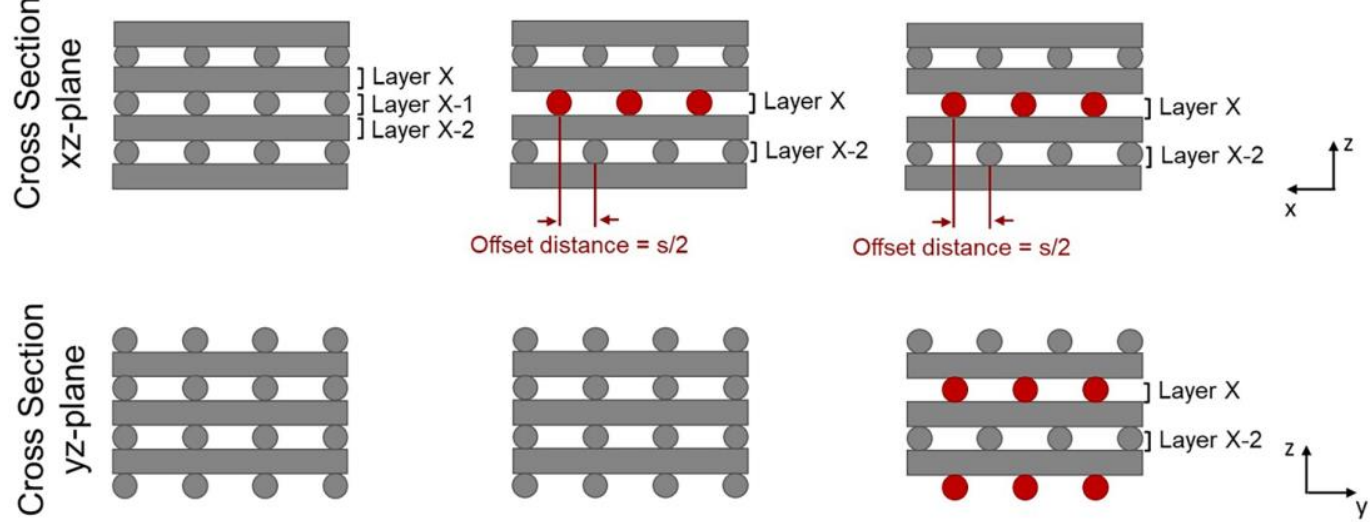

669 Figure 1. (a) Scaffold geometrical features: d, fibre diameter; s, fibre spacing; inter-s,

670 fibre inter-spacing; LT, layer thickness; 1, length of the scaffold; h, height of the scaffold.

671 (b-d) Schematic describing the different filament patterns of the designed scaffolds

672 consisting of a regular orthogonal architecture in the case of (b) the Aligned pattern,

673 whereas offset layers are present only in one plane for (c) the Single Offset pattern or in

674 both planes for (d) the Double Offset pattern. Offset layers are indicated in red.

675

676

677

678

679

680

681 
682
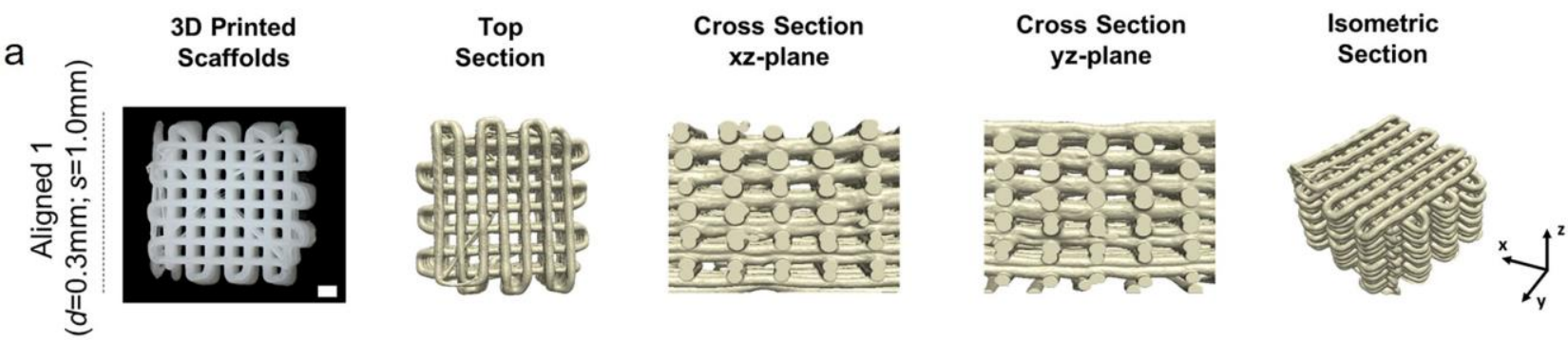

b
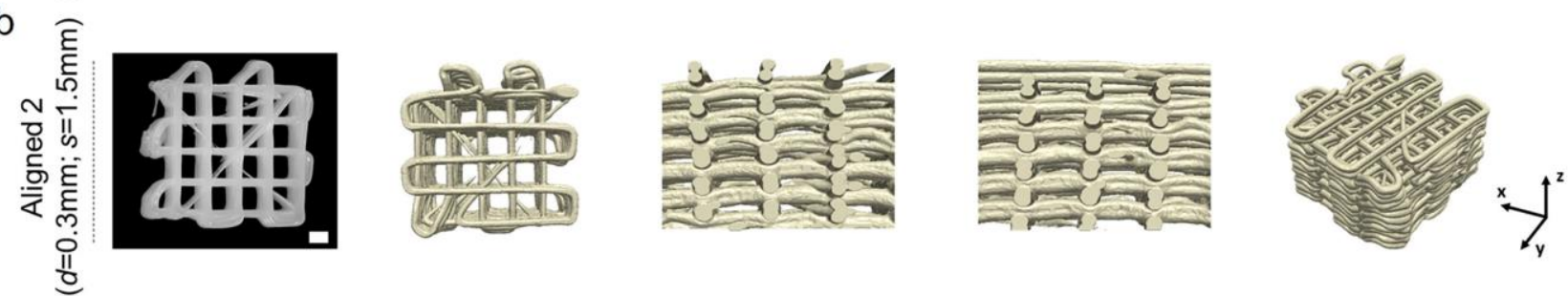

$\circ$
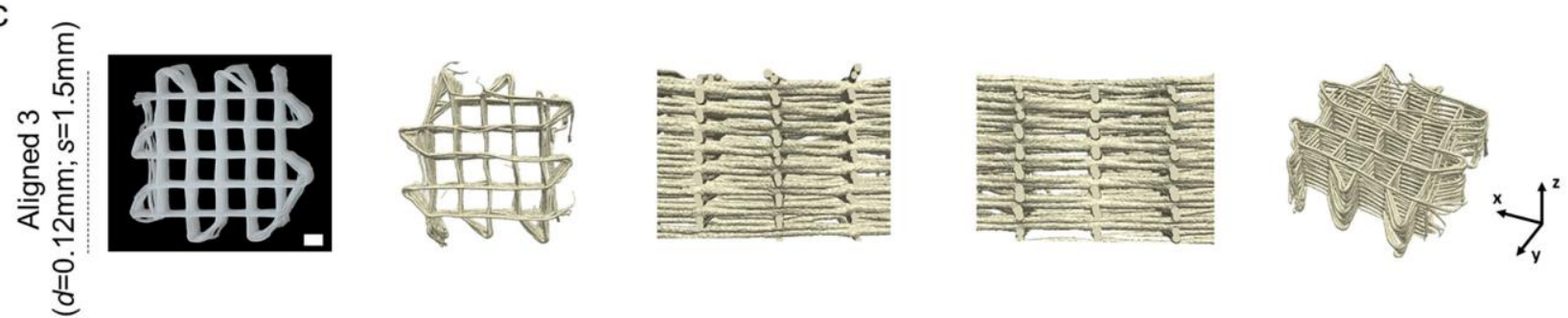

683 Figure 2. Microscopy and micro-CT images of (a) Aligned $1(d=0.3 \mathrm{~mm} ; s=1.0 \mathrm{~mm})$, (b)

684 Aligned $2(d=0.3 \mathrm{~mm} ; s=1.5 \mathrm{~mm})$ and (c) Aligned $3(d=0.12 \mathrm{~mm} ; s=1.5 \mathrm{~mm})$ scaffolds 685 fabricated via 3D printing. Scale bar: $1 \mathrm{~mm}$.

686

687 
a
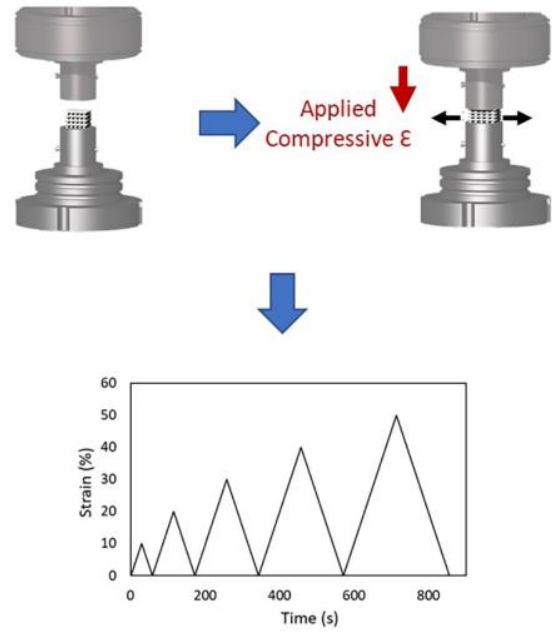

C

Effect of Fibre Spacing

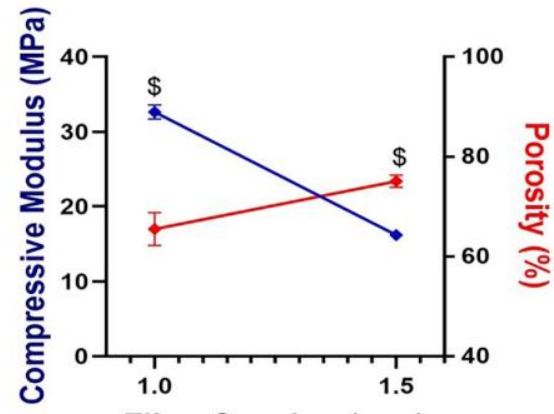

Fibre Spacing $(\mathrm{mm})$

e

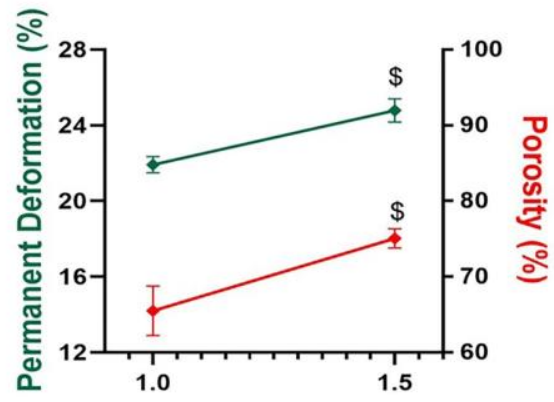

Fibre Spacing $(\mathbf{m m})$ b

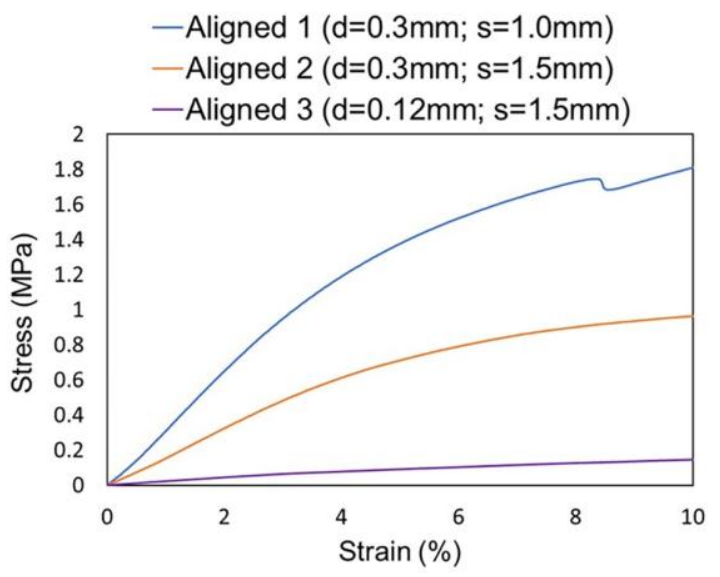

d

Effect of Fibre Diameter

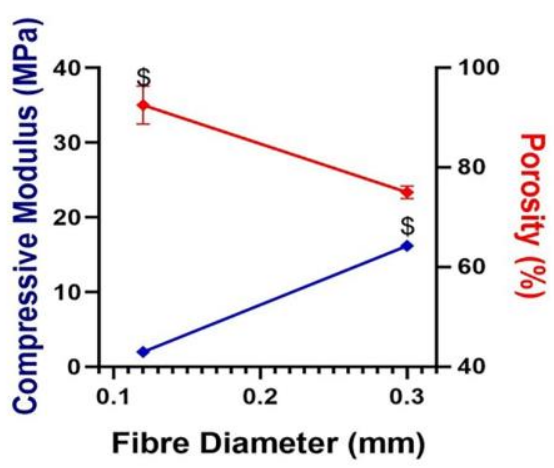

f

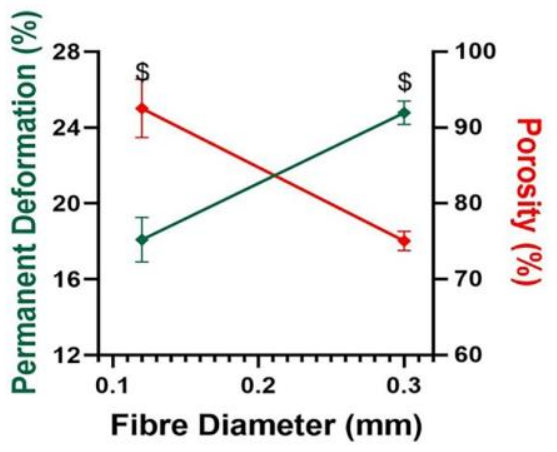

689 Figure 3. (a) Schematic illustration of the mechanical testing set-up and protocol used to

690 perform unconfined cyclic compression tests. (b) Representative stress-strain curves for

691 Aligned $1(d=0.3 \mathrm{~mm} ; s=1.0 \mathrm{~mm})$, Aligned $2(d=0.3 \mathrm{~mm} ; s=1.5 \mathrm{~mm})$ and Aligned $3(d=0.12 \mathrm{~mm}$;

$692 s=1.5 \mathrm{~mm})$ architectures. Effect of modifying (c,e) fibre spacing and (d,f) fibre diameter

693 on porosity, compressive modulus and permanent deformation of 3D-printed PCL

694 constructs. ${ }^{\$} \mathrm{p}<0.01, \mathrm{n}=4$ per group. 


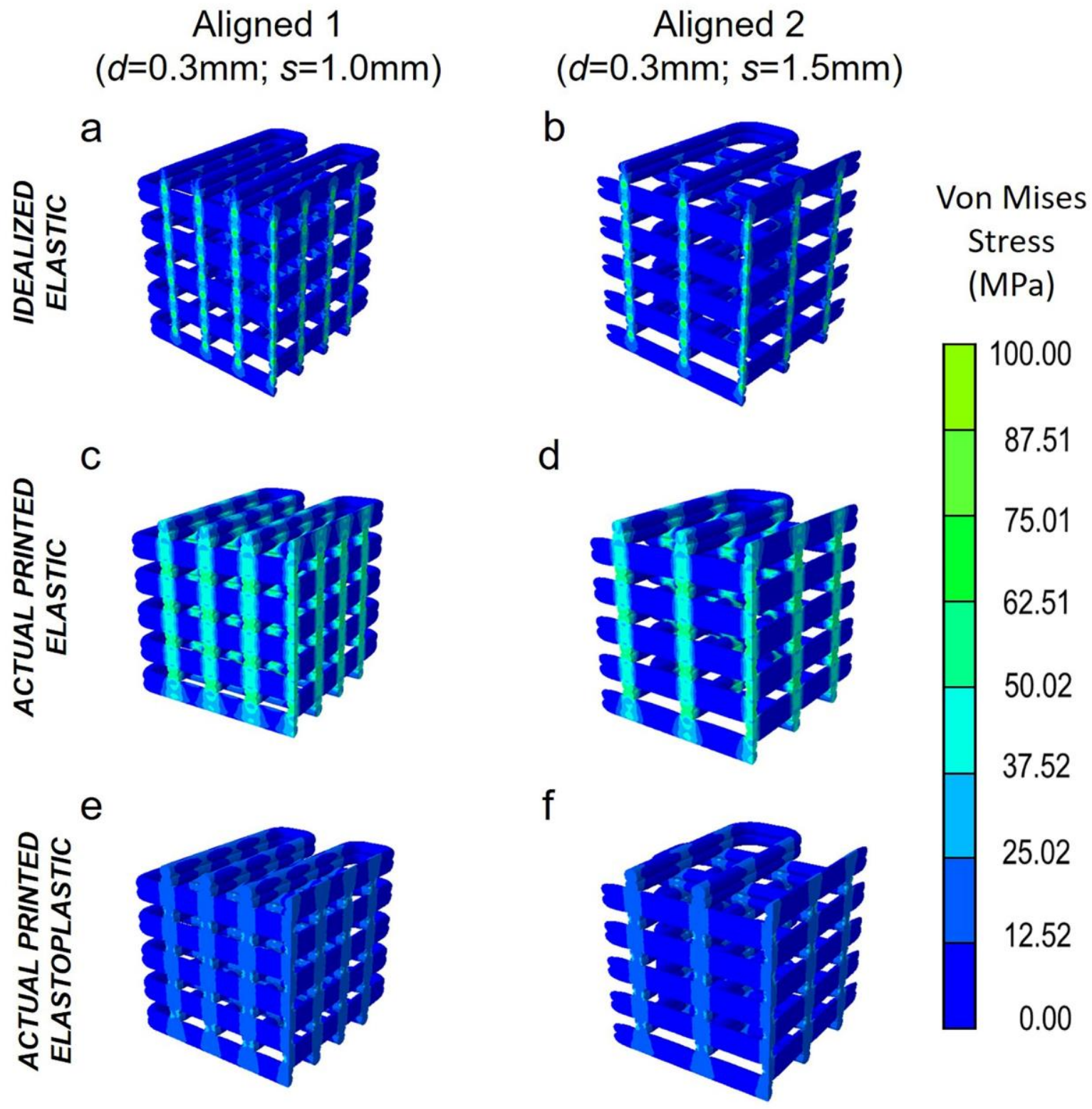

695

696 Figure 4. Comparison of von Mises stress distribution in (a,b) idealized elastic, (c,d)

697 actual printed elastic and (e,f) actual printed elastoplastic models for (a,c,e) Aligned 1

$698(d=0.3 \mathrm{~mm} ; s=1.0 \mathrm{~mm})$ and $(\mathrm{b}, \mathrm{d}, \mathrm{f})$ Aligned $2(d=0.3 \mathrm{~mm} ; s=1.5 \mathrm{~mm})$ designs.

699

700

701 
a

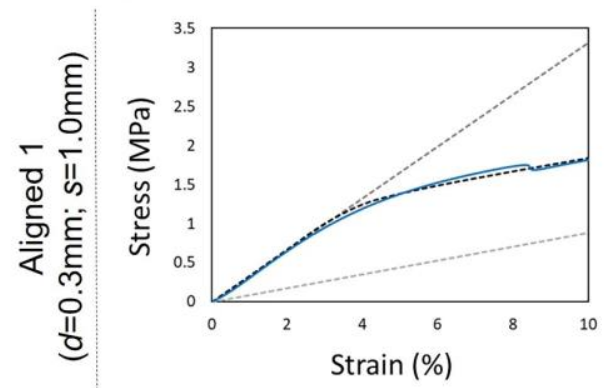

C

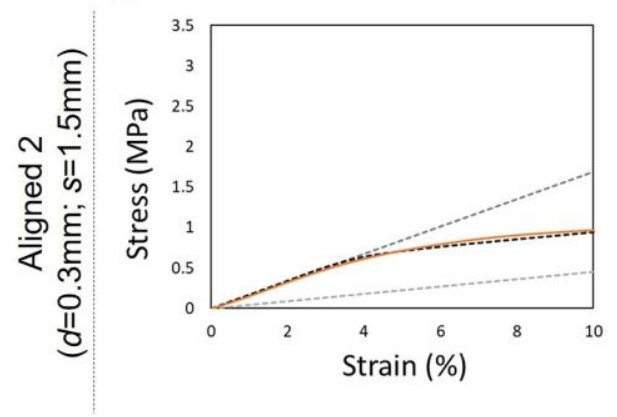

b

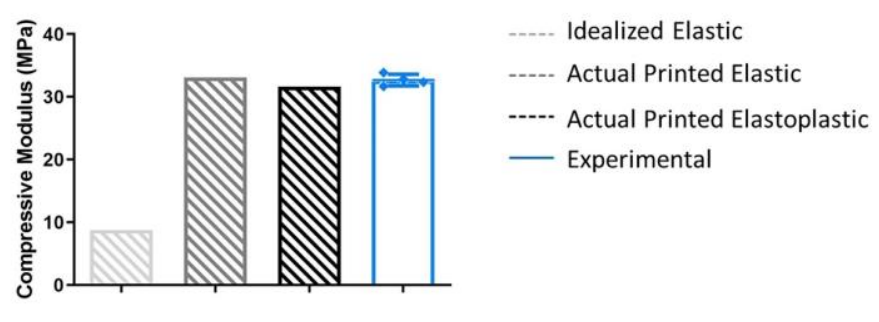

d

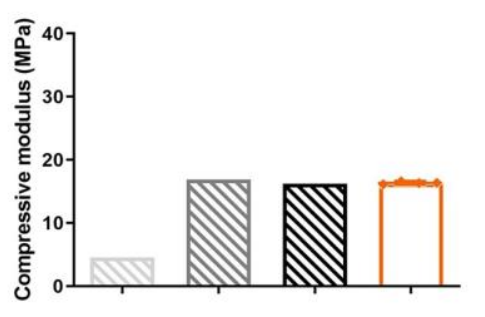

-..-. Idealized Elastic

---- Actual Printed Elastic

---- Actual Printed Elastoplastic

- Experimental

702

Figure 5. (a-d) Predicted (idealized elastic, actual printed elastic and actual printed and Aligned $2(d=0.3 \mathrm{~mm} ; s=1.5 \mathrm{~mm})$ designs. Compression stress-strain diagrams (a,c) and compressive modulus values $(\mathrm{b}, \mathrm{d})$ for $(\mathrm{a}, \mathrm{b})$ Aligned $1(d=0.3 \mathrm{~mm} ; s=1.0 \mathrm{~mm})$ and $(\mathrm{c}, \mathrm{d})$ Aligned $2(d=0.3 \mathrm{~mm} ; s=1.5 \mathrm{~mm})$ structures.

708

709

710

711

712

713

714

715

716 


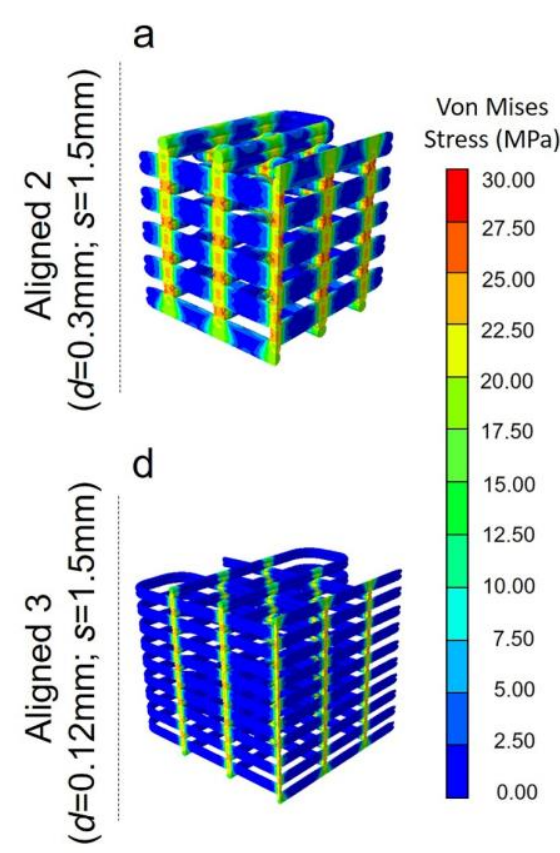

b

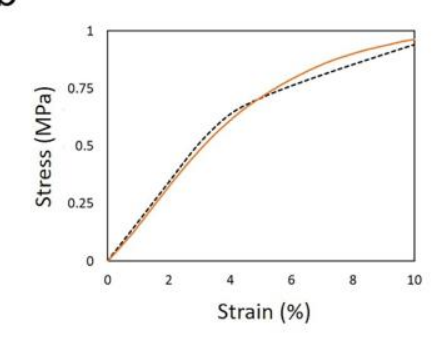

e

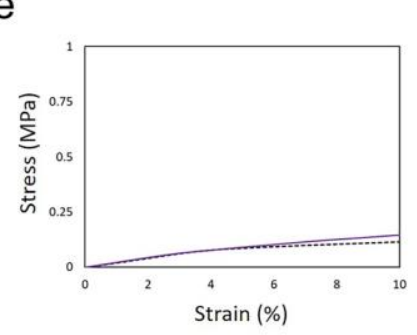

C

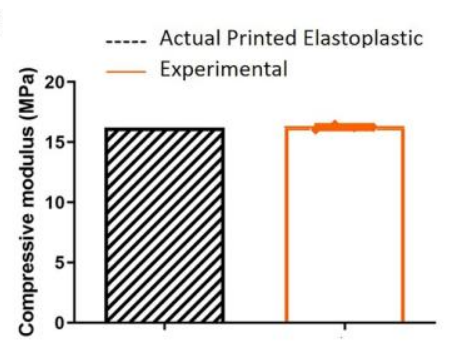

$f$

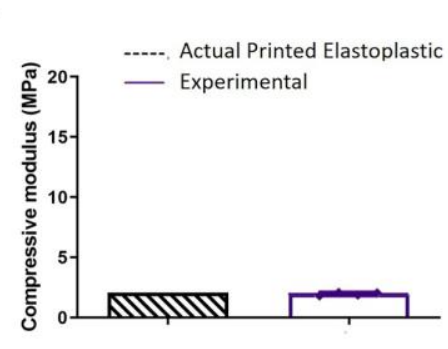

718

719 Figure 6. Comparison of von Mises stress distribution in actual printed elastoplastic models for (a) Aligned $2(d=0.3 \mathrm{~mm} ; s=1.5 \mathrm{~mm})$ and (d) Aligned $3(d=0.12 \mathrm{~mm} ; s=1.5 \mathrm{~mm})$

721 designs. Predicted and experimental (b,e) compression stress-strain diagrams and (c,f)

722 compressive moduli for (b,c) Aligned $2(d=0.3 \mathrm{~mm} ; s=1.5 \mathrm{~mm})$ and $(\mathrm{e}, \mathrm{f})$ Aligned 3 $723(d=0.12 \mathrm{~mm} ; s=1.5 \mathrm{~mm})$ structures. 


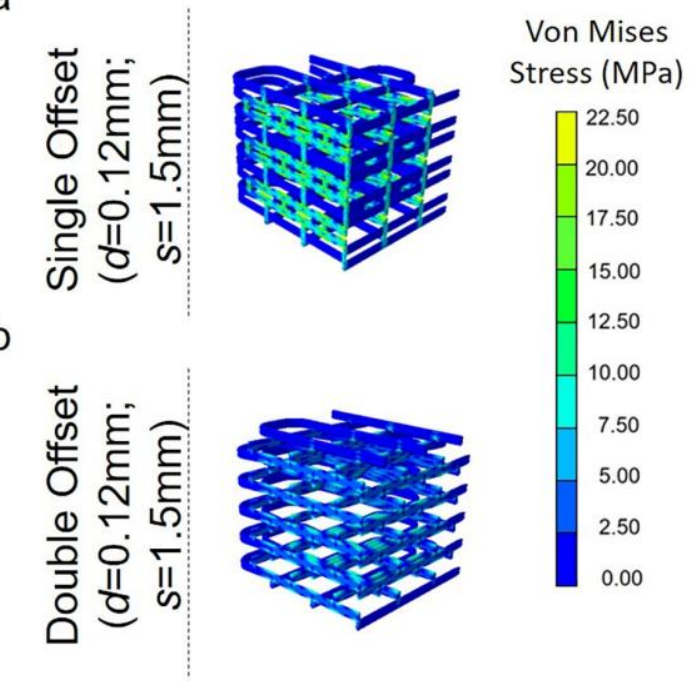

C

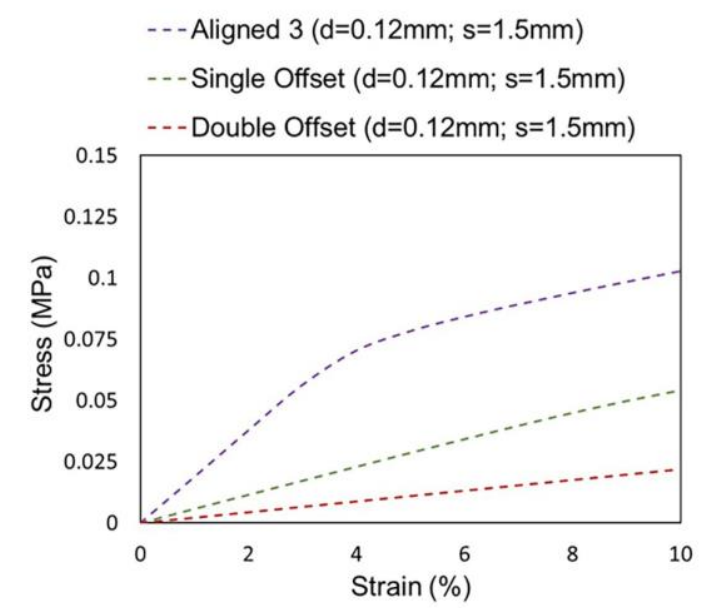

e

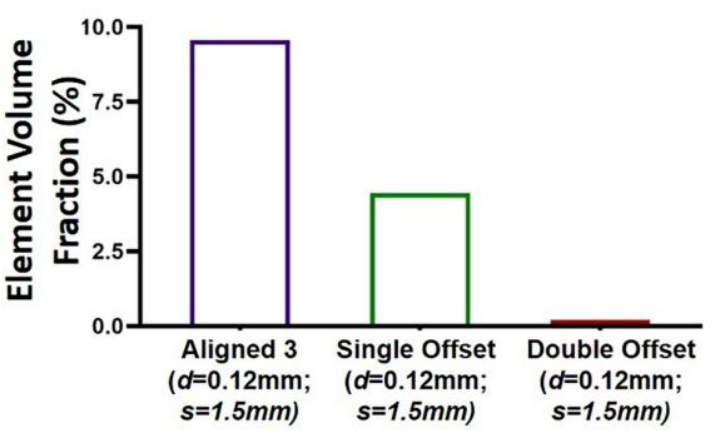

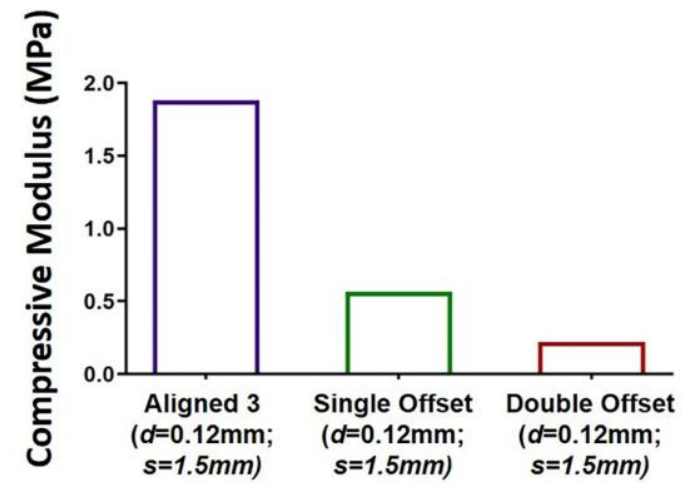

728 Figure 7. Von Mises stress contour plots for (a) Single Offset $(d=0.12 \mathrm{~mm} ; s=1.5 \mathrm{~mm})$ and

(b) Double Offset ( $d=0.12 \mathrm{~mm} ; s=1.5 \mathrm{~mm})$ structures. Computational (c) compression stress-

strain graph, (d) compressive moduli and (e) element volume fraction experiencing

731 stresses greater than $17 \mathrm{MPa}$ (PCL yield stress) comparing Aligned 3 ( $d=0.12 \mathrm{~mm}$;

$732 s=1.5 \mathrm{~mm})$, Single Offset $(d=0.12 \mathrm{~mm} ; s=1.5 \mathrm{~mm})$ and Double Offset $(d=0.12 \mathrm{~mm} ; s=1.5 \mathrm{~mm})$

733 geometries. 

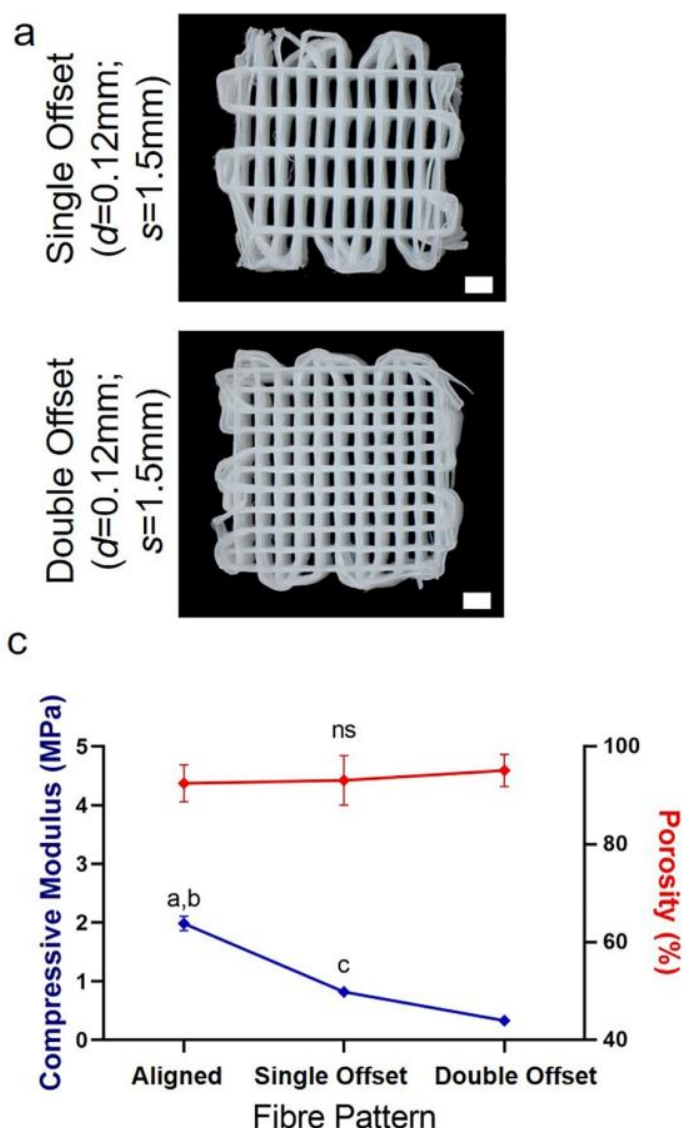

b

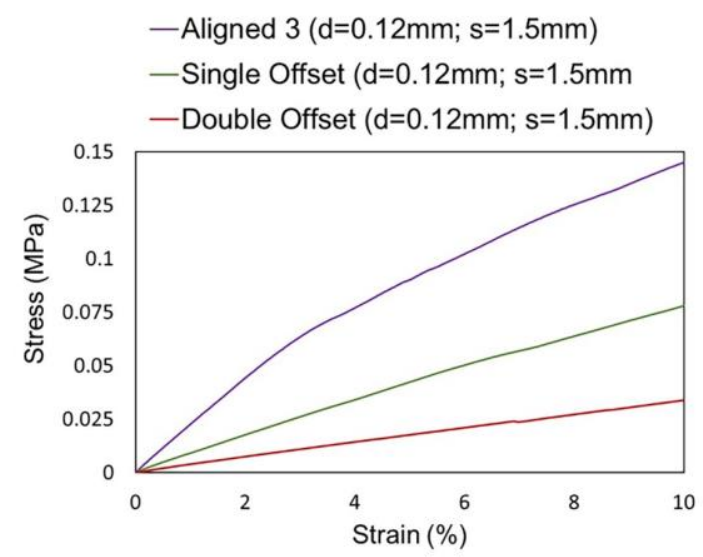

d

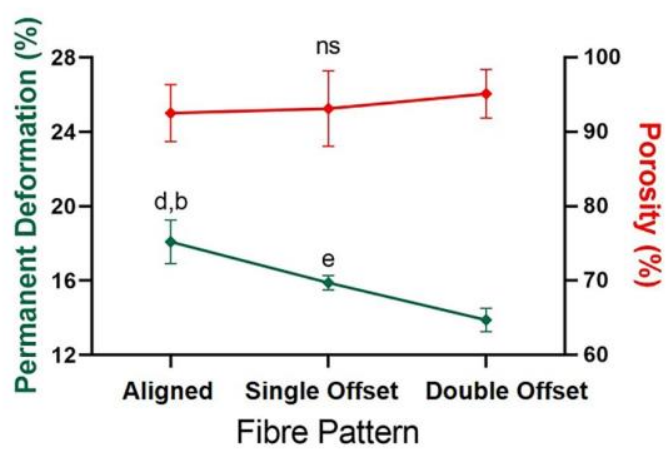

737 Figure 8. (a) Microscopy images of Single Offset ( $d=0.12 \mathrm{~mm} ; s=1.5 \mathrm{~mm})$ (top) and Double Offset $(d=0.12 \mathrm{~mm} ; s=1.5 \mathrm{~mm})$ (bottom) 3D-printed PCL scaffolds; scale bar: $1 \mathrm{~mm}$.

739 Representative experimental stress-strain curves for Aligned $3(d=0.12 \mathrm{~mm} ; s=1.5 \mathrm{~mm})$,

740 Single Offset $(d=0.12 \mathrm{~mm} ; s=1.5 \mathrm{~mm})$ and Double Offset $(d=0.12 \mathrm{~mm} ; s=1.5 \mathrm{~mm})$ architectures.

741 (c,d) Effect of modifying fibre pattern on porosity, compressive modulus and permanent

742 deformation of 3D-printed PCL constructs. ${ }^{a} \mathrm{p}<0.0001$ Aligned $3(d=0.12 \mathrm{~mm} ; s=1.5 \mathrm{~mm}) \mathrm{vs}$.

743 Single Offset $(d=0.12 \mathrm{~mm} ; s=1.5 \mathrm{~mm}),{ }^{\mathrm{b}} \mathrm{p}<0.0001$ Aligned $3(d=0.12 \mathrm{~mm} ; s=1.5 \mathrm{~mm})$ vs.

744 Double Offset $(d=0.12 \mathrm{~mm} ; s=1.5 \mathrm{~mm}),{ }^{c} \mathrm{p}<0.0001$ Single Offset $(d=0.12 \mathrm{~mm} ; s=1.5 \mathrm{~mm})$ vs.

745 Double Offset $(d=0.12 \mathrm{~mm} ; s=1.5 \mathrm{~mm})$ when evaluating the compressive moduli, $\mathrm{n}=4$ per

746 group. ${ }^{\mathrm{d}} \mathrm{p}<0.01$ Aligned $3(d=0.12 \mathrm{~mm} ; s=1.5 \mathrm{~mm})$ vs. Single Offset $(d=0.12 \mathrm{~mm} ; s=1.5 \mathrm{~mm})$,

$747 \mathrm{~b}_{\mathrm{p}}<0.0001$ Aligned $3(d=0.12 \mathrm{~mm} ; s=1.5 \mathrm{~mm})$ vs. Double Offset $(d=0.12 \mathrm{~mm} ; s=1.5 \mathrm{~mm})$, $748{ }_{\mathrm{p}}^{\mathrm{e}}<0.01$ Single Offset $(d=0.12 \mathrm{~mm} ; s=1.5 \mathrm{~mm})$ vs. Double Offset $(d=0.12 \mathrm{~mm} ; s=1.5 \mathrm{~mm})$ when 749 evaluating the permanent deformation, $n=4$ per group. 
a

Aligned 1

$(d=0.3 \mathrm{~mm} ; \mathrm{s}=1.0 \mathrm{~mm})$
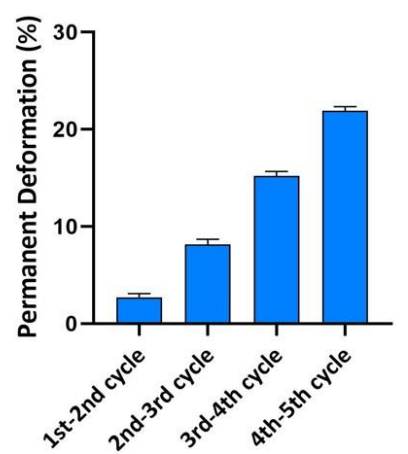

d

Single Offset

$(d=0.12 \mathrm{~mm} ; \mathrm{s}=1.5 \mathrm{~mm})$
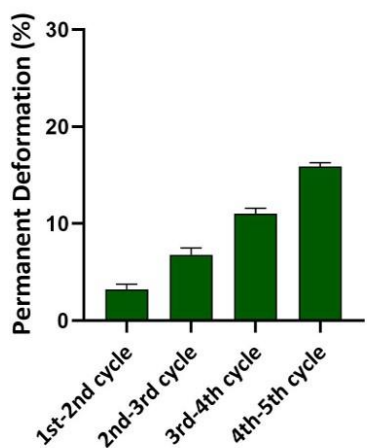

b

Aligned 2

$(d=0.3 \mathrm{~mm} ; s=1.5 \mathrm{~mm})$

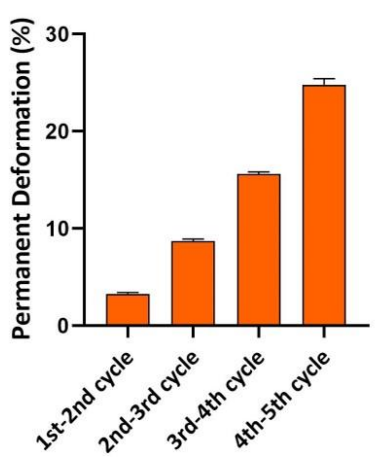

e

$$
\begin{gathered}
\text { Double Offset } \\
(d=0.12 \mathrm{~mm} ; s=1.5 \mathrm{~mm})
\end{gathered}
$$

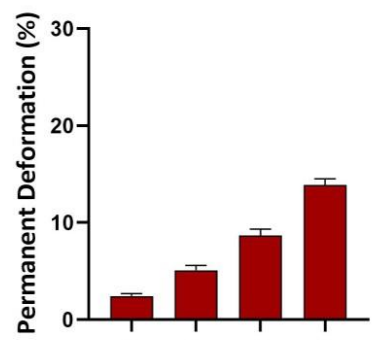

750

751 Double Offset ( $d=0.12 \mathrm{~mm} ; s=1.5 \mathrm{~mm})$ scaffold geometries.

(1)

C Aligned 3

$(d=0.12 \mathrm{~mm} ; \mathrm{s}=1.5 \mathrm{~mm})$

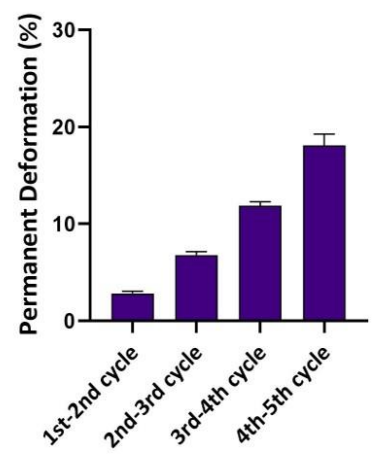

Supplementary Figure 1. Permanent deformation at each applied compressive strain amplitude for (a) Aligned $1(d=0.3 \mathrm{~mm} ; s=1.0 \mathrm{~mm})$, (b) Aligned $2(d=0.3 \mathrm{~mm} ; s=1.5 \mathrm{~mm})$, (c) Aligned $3(d=0.12 \mathrm{~mm} ; s=1.5 \mathrm{~mm})$, (d) Single Offset $(d=0.12 \mathrm{~mm} ; s=1.5 \mathrm{~mm})$ and (e) 
762 Table 1. Summary of FDM printing parameters.

\begin{tabular}{|c|c|c|}
\hline Printing Parameters & $\begin{array}{l}\text { Aligned } 1(d=0.3 \mathrm{~mm} ; s=1.0 \mathrm{~mm}) \\
\text { Aligned } 2(d=0.3 \mathrm{~mm} ; s=1.5 \mathrm{~mm})\end{array}$ & $\begin{array}{c}\text { Aligned } 3(d=0.12 \mathrm{~mm} ; s=1.5 \mathrm{~mm}) \\
\text { Single Offset }(d=0.12 \mathrm{~mm} ; s=1.5 \mathrm{~mm}) \\
\text { Double Offset }(d=0.12 \mathrm{~mm} ; s=1.5 \mathrm{~mm})\end{array}$ \\
\hline Needle (Gauge) & 25 & 30 \\
\hline Layer Thickness (mm) & 0.22 & 0.1 \\
\hline Pressure (MPa) & 0.5 & 0.1 \\
\hline Printing Speed (mm/s) & 4 & 6 \\
\hline Extrusion Speed (revs/m) & 14 & 10 \\
\hline Tank Temperature $\left({ }^{\circ} \mathrm{C}\right)$ & 70 & 86 \\
\hline Needle Temperature $\left({ }^{\circ} \mathrm{C}\right)$ & 70 & 78 \\
\hline
\end{tabular}

763 
Table 2. Idealized geometrical parameters defined for scaffold fabrication versus actual printed geometrical parameters measured after

\begin{tabular}{|c|c|c|c|c|c|c|c|c|c|c|}
\hline \multirow{2}{*}{76} & \multicolumn{2}{|c|}{ Fibre Diameter (mm) } & \multicolumn{2}{|c|}{ Fibre Spacing $(\mathbf{m m})$} & \multicolumn{2}{|c|}{ Fibre Inter-spacing (mm) } & \multicolumn{2}{|c|}{ Layer Fusion (mm) } & \multicolumn{2}{|c|}{ Porosity (\%) } \\
\hline & $\begin{array}{l}\text { Idealized } \\
\text { Geometry }\end{array}$ & $\begin{array}{c}\text { Actual } \\
\text { Printed } \\
\text { Geometry }\end{array}$ & $\begin{array}{l}\text { Idealized } \\
\text { Geometry }\end{array}$ & $\begin{array}{c}\text { Actual } \\
\text { Printed } \\
\text { Geometry }\end{array}$ & $\begin{array}{l}\text { Idealized } \\
\text { Geometry }\end{array}$ & $\begin{array}{c}\text { Actual } \\
\text { Printed } \\
\text { Geometry }\end{array}$ & $\begin{array}{l}\text { Idealized } \\
\text { Geometry }\end{array}$ & $\begin{array}{c}\text { Actual } \\
\text { Printed } \\
\text { Geometry }\end{array}$ & $\begin{array}{l}\text { Idealized } \\
\text { Geometry }\end{array}$ & $\begin{array}{c}\text { Actual } \\
\text { Printed } \\
\text { Geometry }\end{array}$ \\
\hline $\begin{array}{c}\text { Aligned } 1 \\
(d=0.3 \mathrm{~mm} ; \\
s=1.0 \mathrm{~mm})\end{array}$ & 0.26 & $0.307 \pm 0.014$ & 1 & $1.064 \pm 0.106$ & 0.74 & $0.660 \pm 0.017$ & 0 & $0.084 \pm 0.011$ & 80.4 & $67.48 \pm 0.931$ \\
\hline $\begin{array}{l}\text { Aligned } 2 \\
(d=0.3 \mathrm{~mm} ; \\
s=1.5 \mathrm{~mm})\end{array}$ & 0.26 & $0.296 \pm 0.021$ & 1.5 & $1.465 \pm 0.114$ & 1.24 & $1.168 \pm 0.089$ & 0 & $0.076 \pm 0.012$ & 86.2 & $75.05 \pm 1.267$ \\
\hline $\begin{array}{c}\text { Aligned 3 } \\
(d=0.12 \mathrm{~mm} \\
s=1.5 \mathrm{~mm})\end{array}$ & 0.16 & $0.121 \pm 0.015$ & 1.5 & $1.540 \pm 0.061$ & 1.34 & $1.373 \pm 0.025$ & 0 & $0.018 \pm 0.009$ & 91.9 & $92.50 \pm 3.840$ \\
\hline $\begin{array}{l}\text { Single Offset } \\
(d=0.12 \mathrm{~mm} ; \\
\quad s=1.5 \mathrm{~mm})\end{array}$ & 0.16 & $0.127 \pm 0.030$ & 1.5 & $1.526 \pm 0.035$ & 1.34 & $1.392 \pm 0.041$ & 0 & $0.022 \pm 0.012$ & 92.1 & $93.10 \pm 5.081$ \\
\hline $\begin{array}{c}\text { Double Offset } \\
\begin{array}{c}(d=0.12 \mathrm{~mm} ; \\
s=1.5 \mathrm{~mm})\end{array}\end{array}$ & 0.16 & $0.130 \pm 0.023$ & 1.5 & $1.530 \pm 0.092$ & 1.34 & $1.379 \pm 0.103$ & 0 & $0.025 \pm 0.003$ & 92.8 & $95.13 \pm 3.290$ \\
\hline
\end{tabular}


768 Table 3. Elastic and plastic material parameters used for the numerical analysis of PCL

769 scaffolds where E is the Young's modulus; $v$ is the Poisson's ration; $\sigma_{\text {true }}^{y}$ is the true

770 yield stress; $\varepsilon_{p l}^{y}$ is the true plastic yield strain; $\sigma_{\text {true }}^{f}$ is the true stress at failure; $\varepsilon_{p l}^{f}$ is the 771 true plastic strain at failure.

\begin{tabular}{|c|l|l|}
\hline Material & \multicolumn{1}{|c|}{ Material model } & Material Properties \\
\hline PCL & Isotropic elastic & $\begin{array}{l}E=430 \mathrm{MPa} \\
\mathrm{v}=0.3\end{array}$ \\
\hline & Isotropic plastic & $\sigma_{\text {true }}^{y}=17.745 \mathrm{MPa}$ \\
& & $\varepsilon_{p l}^{y}=0$ \\
& & $\sigma_{\text {true }}^{f}=113.39 \mathrm{MPa}$ \\
& & $\varepsilon_{p l}^{f}=1.3316$ \\
\hline
\end{tabular}

772 